\title{
Gravitational instability of planetary gaps and its effect on orbital migration
}

\author{
Min-Kai Lin and Ryan Cloutier \\ Canadian Institute for Theoretical Astrophysics, 60 St. George Street, Toronto, ON, M5S 3H8, \\ Canada \\ email: mklin924@cita.utoronto.ca, cloutier@cita.utoronto.ca
}

\begin{abstract}
Gap formation by giant planets in self-gravitating disks may lead to a gravitational edge instability (GEI). We demonstrate this GEI with global 3D and 2D self-gravitating diskplanet simulations using the ZEUS, PLUTO and FARGO hydrodynamic codes. High resolution 2D simulations show that an unstable outer gap edge can lead to outward migration. Our results have important implications for theories of giant planet formation in massive disks.
\end{abstract}

Keywords. hydrodynamics, instabilities, methods: numerical, planetary systems: protoplanetary disks, planets and satellites: formation

\section{Introduction: the gravitational edge instability}

Models of giant planet formation in self-gravitating protoplanetary disks may involve gap-opening in massive disks. However, gaps or edges in self-gravitating disks are potentially unstable to a gravitational edge instability (GEI) associated with local potential vorticity maxima (Meschiari \& Laughlin 2008; Lin \& Papaloizou 2011).

We confirm the GEI for planet-induced gaps with global disk-planet simulations. We consider a locally isothermal disk with constant aspect-ratio $h=0.05$ and Keplerian Toomre parameter $Q_{k}=1.5$ at the outer disk boundary $r_{\text {out }}=2.5 r_{p}$, where $r_{p}$ is the fixed orbital radius of a planet with mass $M_{p}=10^{-3} M_{*}$ and $M_{*}$ is the central star mass. We simulated this self-gravitating disk-planet system in 3D using the ZEUS-MP code (Hayes et al. 2006) and PLUTO code (Mignone et al. 2007); and in 2D using the FARGO code (Baruteau \& Masset 2008). The 3D simulations are performed in spherical polar co-ordinates covering two vertical scale-heights.

Fig. 1 shows the outer gap edge develops a spiral instability with azimuthal wavenumber $m=2$ (for 3D runs one of the spirals coincides with the planet wake in the snapshot). The spirals extend close to $r_{p}$. In fact, the GEI co-rotation radius is just inside a giant planet's co-orbital region (Lin \& Papaloizou 2011). So the GEI supplies fluid onto horseshoe orbits ahead of the planet, which provides a positive disk-on-planet torque (Lin \& Papaloizou 2012).

\section{Instability-induced outward migration and implications}

We also performed 2D FARGO simulations with different $M_{p}$ where the planet is allowed to migrate, so that $r_{p}=r_{p}(t)$. Fig. 2 shows that for $t \lesssim 100$ orbits, outward migration proceeds at a faster rate with increasing $M_{p}$, because the GEI becomes stronger. Eventually, a GEI spiral-planet scattering event triggers rapid outward type III migration. In the context of the disk fragmentation model for wide-orbit giant planet formation (Vorobyov 2013), this suggests that gap-opening may not be sufficient to maintain giant planets on a fixed orbital radius beyond a few tens of orbits. 

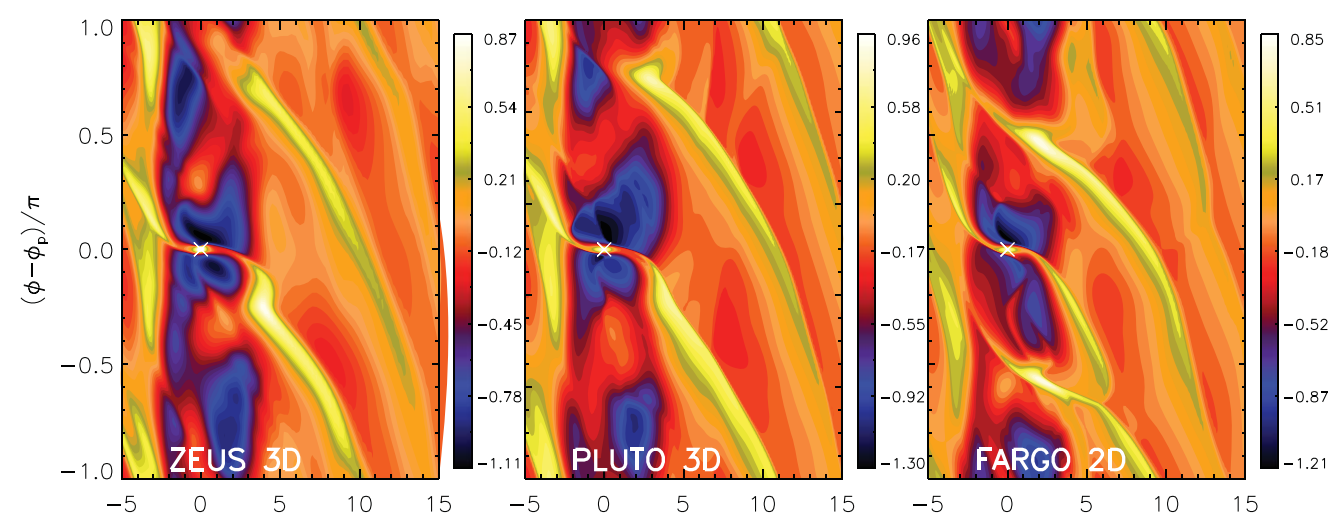

Figure 1. Gravitational edge instability of a planetary gap. The logarithmic midplane density perturbation is shown for 3D runs (left, middle) and the logarithmic surface density perturbation is shown for the 2D run (right). Snapshots are taken at 15 (10) orbits after the planet is introduced into the 3D (2D) disk. Horizontal axis are in units of Hill radii. The planet is at the origin. The left and right simulations are taken from Lin (2012) and Cloutier \& Lin (2013), respectively.

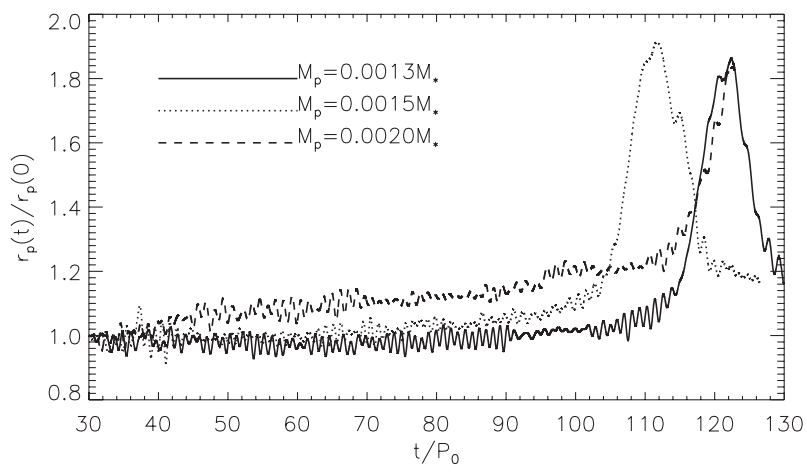

Figure 2. Orbital migration of giant planets when the outer gap edge is gravitationally unstable. Here, $P_{0}$ is the orbital period at the planet's initial orbital radius. Adapted from 2D FARGO simulations presented in Cloutier \& Lin (2013).

Acknowledgments. Several computations were performed on the GPC supercomputer at the SciNet HPC Consortium. SciNet is funded by: the Canada Foundation for Innovation under the auspices of Compute Canada; the Government of Ontario; Ontario Research Fund - Research Excellence; and the University of Toronto.

\section{References}

Baruteau, C. \& Masset, F. 2008, ApJ, 678, 483

Cloutier, R. \& Lin, M.-K. 2013, MNRAS, 434, 621

Hayes, J. C., Norman, M. L., Fiedler, R. A., Bordner, J. O., Li, P. S., Clark, S. E., ud-Doula, A., \& Mac Low, M. 2006, ApJS, 165, 188

Lin, M.-K. 2012, MNRAS, 426, 3211

Lin, M.-K. \& Papaloizou, J. C. B. 2011, MNRAS, 415, 1445

Lin, M.-K. \& Papaloizou, J. C. B. 2012, MNRAS, 421, 780

Meschiari, S. \& Laughlin, G. 2008, ApJL, 679, L135

Mignone, A., Bodo, G., Massaglia, S., Matsakos, T., Tesileanu, O., Zanni, C., \& Ferrari, A. 2007, ApJS, 170, 228

Vorobyov, E. I. 2013, A\&A, 552, A129 